\title{
Distribution of growth directions in meadows of clonal plants
}

\author{
D. Ruiz-Reynés* and D. Gomila \\ IFISC (CSIC-UIB), Instituto de Física Interdisciplinar y Sistemas Complejos, Campus Universitat de les Illes Balears, \\ E-07122 Palma de Mallorca, Spain
}

(Received 27 June 2019; revised manuscript received 18 September 2019; published 18 November 2019)

\begin{abstract}
Clonal growth plants are abundant in both terrestrial and marine ecosystems. Some marine species are particularly important since they provide essential ecosystem services in the shores of all continents except Antarctica. For the appropriate modelization of clonal growth the discretization of the growth direction angle has to be carefully treated to correctly describe the dynamics of the meadow. Specifically, determining the minimum number of growth directions is important to maximize the efficiency of numerical simulations. We show that the presence of neutral modes in the growth direction angle tends to make the distribution of the growth directions uniform in the presence of noise, allowing us to choose the minimal number of discrete angles compatible with the branching. We also show that the formation of spatial patterns induces small differences in the population density within these different growth directions.
\end{abstract}

DOI: 10.1103/PhysRevE.100.052208

\section{INTRODUCTION}

Clonal growth plants are present in a wide variety of both terrestrial and marine ecosystems around the globe. Particularly, seagrasses range among the most productive on Earth [1] and form the basis of many marine habitats. Of special interest are those ecosystems formed by Posidonia oceanica. This endemic plant of the Mediterranean Sea, which grows a few centimeters per year, forms large meadows promoting biodiversity and providing valuable services, such as shoreline protection, water filtration and oxygenation, or intense $\mathrm{CO}_{2}$ sequestration [2,3]. Despite its importance for the conservation of the ecosystem this marine plant is one of the most threatened [4], with several causes contributing to its global decline $[3,4]$. The vulnerability against anthropogenic factors and the difficulties that present the restoration of the habitat, essentially because of the temporal scales involved in the growth, make the understanding of the spatial distribution of the meadow a key factor for its conservation.

From this perspective we have recently proposed a model for the description of clonal growth plants [5]. This model describes quantitatively the development of large meadows based on clonal growth rules. On the one hand, previous models based on random walks, diffusion-limited-aggregation or agent-based models [6-11] usually describe clonal growth with greater detail but at smaller spatial scales. On the other hand, models suitable for a landscape description [12-14] use diffusion to reproduce the advance of plants without considering the intrinsic features of clonal spreading. Thus, the model in Ref. [5] bridges the gap between these two approaches describing clonal spreading and long-range spatial interactions simultaneously. With this approach the spatial organization of the plant is explained as a self-organization process which gives rise to pattern formation.

\footnotetext{
*druiz@ifisc.uib-csic.es
}

Despite the description focusing on the spatial distribution of the meadow in two dimensions, which is a good approximation to describe pattern formation on the sea bottom, the model takes into account also the direction of growth of the apices. This fact includes an additional dimension, namely, the angle corresponding to the direction of growth, which is necessary to describe the branching process of the plant. To simplify the tackling of the 3D problem, the angle of the direction of growth is discretized and only a reduced number of directions are considered. In the case of $P$. oceanica, whose branching angle is approximately $45^{\circ}$, the minimum number of directions is 8 , consistent with the branching angle, such that when apices growing in one specific direction branch, the new apices contribute to a direction already taken into account in the model. This situation is, however, artificial and in principle does not describe properly the case where the branching angle is not exactly an integer fraction of $2 \pi$ or if there are fluctuations in the direction of growth. Heterogeneous distributions of the density in the growth direction would neither be well described by a discretization with only a few number of growth directions. Therefore, the understanding of the distribution of growth directions is necessary for a correct modelization and numerical simulation of clonal growth plants.

Here we investigate the possibility of nonhomogeneous growth direction distributions in the apices population. We focus on the particular case of branching angle $\phi_{b}=45^{\circ}$, which corresponds to the case of P.Oceanica but it is not limited to this species [15]. We show that there are neutral modes associated to nonhomogeneous distributions which are periodic with the branching angle. These neutral modes appear due to the breaking of the rotational symmetry in the system by solutions which are not homogeneous in the growth direction. Under the influence of noise these neutral modes tend to restore the symmetry bringing the system back to a homogeneous distribution in the growth direction, thus, justifying using only the minimum number of discrete 
directions. Besides, spatial patterns induce small modulations in the distribution of growth with the periodicity $2 \pi$, which are not neutral and are expected to be robust against noise.

\section{THE ABD MODEL}

The advection-branching-death (ABD) model describes the evolution of the spatial density of shoots $n_{s}(\vec{r}, t)$ and apices $n_{a}(\vec{r}, \phi, t)$ growing in the direction indicated by the angle $\phi$, at position $\vec{r}=(x, y)$ and time $t$ [5]:

$$
\begin{gathered}
\partial_{t} n_{a}(\vec{r}, \phi, t) \\
=-\omega_{d} n_{a}(\vec{r}, \phi, t)-\vec{v}(\phi) \cdot \vec{\nabla} n_{a}(\vec{r}, \phi, t) \\
\quad+\frac{\omega_{b}}{2}\left[n_{a}\left(\vec{r}, \phi+\phi_{b}, t\right)+n_{a}\left(\vec{r}, \phi-\phi_{b}, t\right)\right] \\
\partial_{t} n_{s}(\vec{r}, t)=-\omega_{d} n_{s}(\vec{r}, t)+\frac{v}{\rho} \int_{0}^{2 \pi} n_{a}(\vec{r}, \phi, t) d \phi .
\end{gathered}
$$

There are three main mechanisms determining the evolution of the apices and shoots implemented in the right-hand side of Eqs. (1) and (2). First, living shoots have a typical lifetime depending on external factors and the presence of neighboring shoots, resulting in an overall per capita mortality rate $\omega_{d}$, which affects both shoots and apices. Second, an apex grows elongating the rhizome in a fixed horizontal direction with a velocity $v$, leaving new shoots behind which are separated along the rhizome by a typical distance $\rho$. This mechanism results in an advection term in Eq. (1), where the advection velocity vector is given by $\vec{v}=(v \cos \phi, v \sin \phi)$, and an integral term in Eq. (2) accounting for the shoots left behind by the apices growing in all directions. Last, the growing apices develop new branches at a rate $\omega_{b}$. These branches grow in a horizontal direction forming an angle $\phi_{b}$ with the original rhizome from which they bifurcate. This couples "nonlocally" apices growing in different directions as indicated by the last term of Eq. (1).

The mortality $\omega_{d}$ includes external effects and a number of interaction mechanism:

$$
\omega_{d}\left(n_{t}\right)=\omega_{d 0}+\iint \mathcal{K}\left(\vec{r}-\vec{r}^{\prime}\right)\left(1-e^{-a n_{t}\left(\vec{r}^{\prime}\right)}\right) d \vec{r}^{\prime}+b n_{t}^{2},
$$

where $n_{t}(\vec{r}, t)=n_{s}(\vec{r}, t)+\int_{0}^{2 \pi} n_{a}(\vec{r}, \phi, t) d \phi$ is the total number of plants corresponding to the sum of apices growing in all directions and shoots. The first term $\omega_{d 0}$ is the death rate of a single isolated shoot, i.e., in the absence of any neighboring shoots. The second term is an integral term that accounts for interactions across space, in other words, shoots at a given position can affect the growth in a neighborhood weighted by the kernel $\mathcal{K}\left(\vec{r}-\vec{r}^{\prime}\right)$. The last term is a local nonlinear term which saturates the density of shoots preventing an unlimited growth, $b$ being the parameter that controls the maximum value of the density.

The kernel $\mathcal{K}$ is composed by two normalized Gaussian functions $\mathcal{G}$ with different amplitudes $\left(\kappa, \omega_{d 0}>0\right)$ and widths $\left(\sigma_{\kappa}, \sigma_{0}\right)$ :

$$
\mathcal{K}(\vec{r})=\kappa \mathcal{G}\left(\sigma_{\kappa}, \vec{r}\right)-\omega_{d 0} \mathcal{G}\left(\sigma_{0}, \vec{r}\right)
$$

The first term in the right-hand side of Eq. (4) accounts for all the competitive effects since it increases the mortality rate, while the second for the facilitative effects. The ranges of the

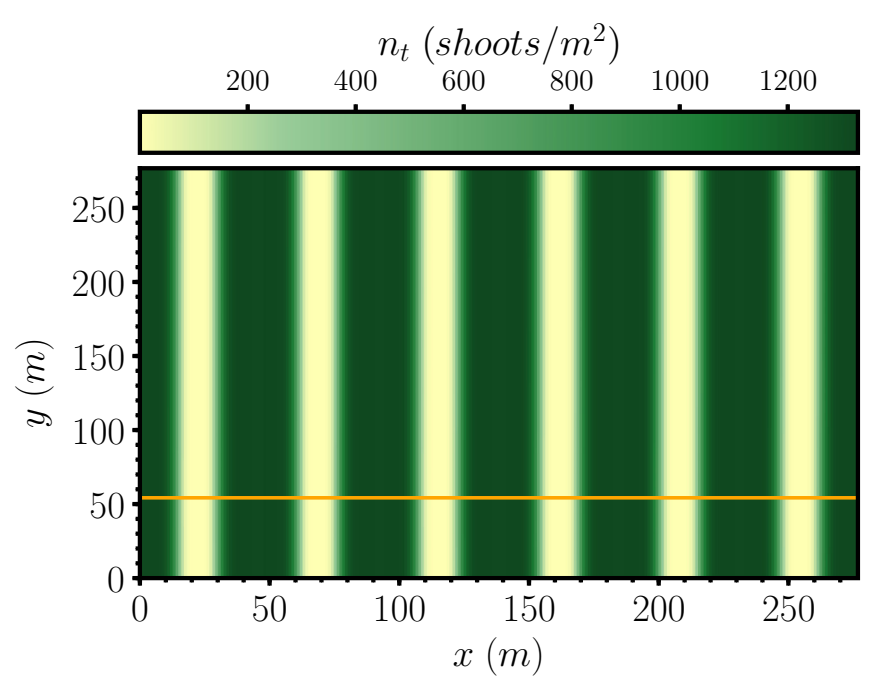

FIG. 1. Stable solution showing a pattern of stripes with wave number $q_{x, c}=0.1357 \mathrm{rad} / \mathrm{m}$. The total density $n_{t}$ is shown. Here $\omega_{b}=0.06$ year $^{-1}, \omega_{d 0}=0.0846$ year $^{-1}$. The orange line indicates the transect at $y$ constant used in other figures. Other parameters: $v=6.11 \mathrm{~cm} /$ year, $\rho=2.87 \mathrm{~cm}, \phi_{b}=45^{\circ}, b=1.25 \mathrm{~cm}^{4}$ year $^{-1}$, $\kappa=0.048$ year $^{-1}, \sigma_{\kappa}=1629.3 \mathrm{~cm}, a=27.38 \mathrm{~cm}^{2}, \sigma_{0}=203.7 \mathrm{~cm}$.

competitive and facilitative interactions are given by $\sigma_{\kappa}$ and $\sigma_{0}$, respectively.

In terms of the ratio $\omega_{d 0} / \omega_{b}$ one can identify different stable states. On the one hand, the homogeneous meadow prevails if branching rate is much bigger than mortality, $n_{t}^{*}$ being the stationary value of the total density of shoots. On the other hand, if mortality is much bigger than branching, then the only possible solution is bare soil. For intermediate values $\left(\omega_{d 0} / \omega_{b} \sim 1\right)$, the influence of the nonlocal interaction becomes noteworthy and, as a result, feedbacks across space develop modulations until a spatial pattern forms. Three basic patterns emerge in the following order increasing the mortality: negative hexagons, stripes (Fig. 1), and positive hexagons [5].

Clonal growth mechanisms are parametrized through direct plant measurements of $\omega_{b}, \phi_{b}, v$, and $\rho$, which are well established for different species of clonal plants $[6,15]$. The branching process determines the temporal scale, more precisely it is given by $\omega_{b}$, which can be interpreted as a birth term. The branching angle, which is the relevant parameter controlling the growth in different directions, will be studied in the next section. Besides, the velocity of growth of the rhizome has a major role in determining the velocity of spreading of the meadow and $\rho$ essentially changes the relation between the density of apices and shoots. In addition, the values of the parameters $\omega_{d 0}, \kappa, a, b, \sigma_{\kappa}$, and $\sigma_{0}$ determining the dependence of mortality with density and nonlocal spatial interactions are obtained using measurements of the density according to experimental evidence. More details can be found in Ref. [5]. The value of the nonlocal competition range $\sigma_{\kappa}$, which essentially determines the wavelength of the pattern, have been chosen without loss of generality smaller than its original value in Ref. [5] for numerical convenience. In the following, if not specified otherwise, all parameters are fixed as described in the caption of Fig. 1. 


\section{DISTRIBUTION OF GROWTH DIRECTIONS IN THE SPATIALLY HOMOGENEOUS SOLUTIONS}

In addition to the spatial distribution of the population density, the ABD model accounts also for the direction of growth of the apices. This direction is given by the angle $\phi$. Two terms depend explicitly on this angle: the branching and the advection. The main parameter that determines the behavior of the system from the perspective of the angular distribution is the branching angle $\phi_{b}$. For the case of $P$. Oceanica $\phi_{b}$ is $49^{\circ}$. Other clonal plants have different branching angles, most of them in the range $30-60^{\circ}$ [15]. From a computational point of view it is, however, much more simple to consider branching angles commensurable with $2 \pi$, such that only a few discrete directions can be taken into account consistently. For the particular case of P. Oceanica analyzed here, the branching angle is chosen $\phi_{b}=45^{\circ}\left(\phi_{b}=\pi / 4\right)$. Thus, it is possible to describe the system numerically with only eight growth directions instead of using a denser grid. Nevertheless, this minimal discretization is not compatible with solutions modulated in the angle with an azimuthal wave number $q_{\phi}>4$. It is therefore crucial to determine the relevance of such solutions in the dynamics of the full system to determine the reliability of the results obtained with only a small number of growth directions. The alternative approach is to consider a dense grid in the angle. For instance, considering 100 growth directions in the case $\phi_{b}=49^{\circ}$, the closest value of the angle compatible with the discretization to $49^{\circ}$ is $50.4^{\circ}$ which is not commensurable with $2 \pi$ and therefore the branching will distribute the density of apices evenly among all the directions. Thus, the exact value of $\phi_{b}$ is better described by a discretization with higher resolution, however, the computational cost increases dramatically. As it is shown in the next sections, the results are equivalent using the closest commensurable branching angle $\left(45^{\circ}\right)$ instead of the exact branching angle $\left(49^{\circ}\right)$ and numerical efficiency is improved.

For a homogeneous solution in space the total density of apices in the stationary state, $N_{a}^{*}=\int_{0}^{2 \pi} n_{a}^{*}(\phi) d \phi$, is related to the total density $n_{t}^{*}$ by $N_{a}^{*}=\rho \omega_{b} /\left(v+\rho \omega_{b}\right) n_{t}^{*}$. In the stationary limit the distribution in the angle $n_{a}^{*}(\phi)$ is not necessary homogeneous, which in general can be written as $n_{a}^{*}(\phi)=$ $\sum_{q_{\phi}} b_{q_{\phi}}^{*} e^{i q_{\phi} \phi}$. Thus, by means of a linear stability analysis one can study the dynamics of perturbations of the form $\delta n_{s}=n_{s}-n_{s}^{*}$ and $\delta n_{a}(\phi)=n_{a}(\phi)-n_{a}^{*}(\phi)=\sum_{q_{\phi}} \delta b_{q_{\phi}} e^{i q_{\phi} \phi}$, with $q_{\phi}=0,1,2,3 \ldots$, around the homogeneous states in space, for both the zero and the populated solutions. For the stationary solutions homogeneous in the angle and, in the commensurable case, those with period $\phi_{b}$, the linear problem for the perturbations reads

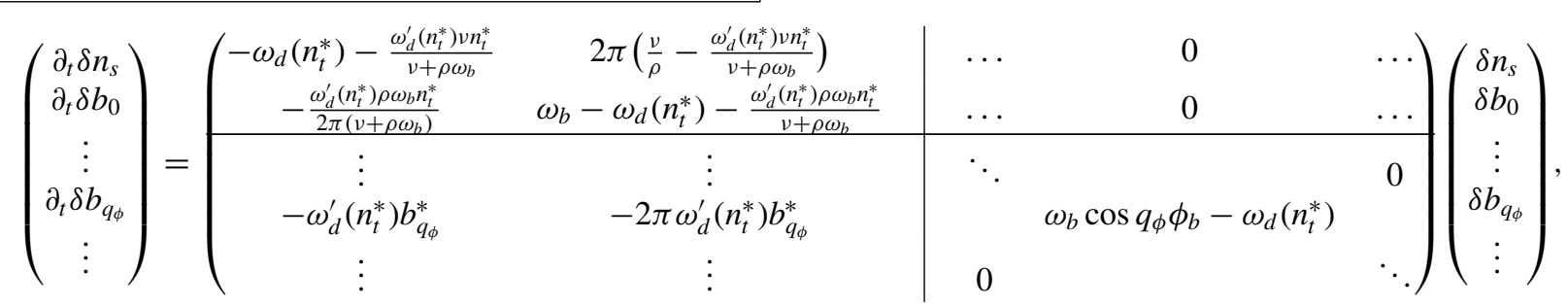

where $\omega_{d}^{\prime}\left(n_{t}^{*}\right)=\left(\kappa-\omega_{d 0}\right) a e^{-a n_{t}^{*}}+2 b n_{t}^{*}$. Thus, the homogeneous components $\delta n_{s}$ and $\delta b_{0}$ are decoupled from $\delta b_{q_{\phi}}$ with $q_{\phi} \geqslant 1$, and the growth rate of such modulations is given in adimensional units by

$$
\lambda\left(q_{\phi}\right)=\cos \left(q_{\phi} \phi_{b}\right)-\omega_{d}^{*},
$$

where $\lambda\left(q_{\phi}\right)$ corresponds to the eigenvalue of the mode with azimuthal wave number $q_{\phi}$. $\omega_{d}^{*}$ corresponds to the stationary and homogeneous value $\omega_{d}^{*}=\omega_{d}\left(n_{t}^{*}\right) / \omega_{b}$. For the unpopulated solution $\omega_{d}^{*}=\omega_{d 0} / \omega_{b}$, and for the populated one $\omega_{d}^{*}=1$.

For $n_{t}^{*}=0$, if the branching rate is smaller than the mortality rate, then all modes have negative real eigenvalues and any small perturbation decays. In the opposite case $\left(\omega_{b}>\omega_{d 0}\right)$, two cases must be considered. If the branching angle is incommensurable with $2 \pi, \cos \left(q_{\phi} \phi_{b}\right)$ is equal to one only for $q_{\phi}=$ 0 , and therefore at $\omega_{b}=\omega_{d 0}$ only the homogeneous mode with eigenvalue $\lambda=1-\omega_{d}^{*}$ becomes unstable, leading above threshold to a solution homogeneous in the angle $\phi$; i.e., all directions of growth are equally populated. On the contrary, if the branching angle is commensurable with $2 \pi, \phi_{b}=2 \pi / m$ $\left(m=8\right.$ in the case of $\left.45^{\circ}\right)$, at $\omega_{b}=\omega_{d 0}$ all modes with $q_{\phi, n}=$ $n m$, with $n=0,1,2,3 \ldots$ become simultaneously unstable. Thus, above threshold, the solution bifurcating at $\omega_{b}=\omega_{d 0}$, which is homogeneous in space but not necessarily in the angle, can be written as $n_{a}^{*}(\phi)=\frac{N_{a}^{*}}{2 \pi}+\sum_{n} b_{q_{\phi, n}} e^{i q_{\phi, n} \phi}$. Any set of arbitrary values of $b_{q_{\phi, n}}$ will be a solution provided the total density of apices $N_{a}^{*}$, given by $2 \pi b_{0}$, takes the proper value determined by $n_{t}^{*}$. We recall also that neither $n_{a}$ nor $n_{s}$ can take negative values. This degeneracy comes from the fact that the only coupling in the angle space is due to the branching, and in the commensurable case, given a direction, the branching couples only a discrete set of $m$ directions separated by $\phi_{b}$. Thus, any arbitrary combination of sets of $m$ directions with the same density separated by $\phi_{b}$, will be a solution, provided the total density sums $n_{t}^{*}$. Therefore, the populated solution homogeneous in space appearing above threshold, depending on the initial conditions, will not necessarily be homogeneous in the angle if the system is deterministic. The effects of noise will be considered later.

The stability of the populated solution is also different in the two cases. When $\phi_{b}$ is incommensurable with $2 \pi$ the solution is homogeneous in the angle and all modes are stable. The meadow grows equally in all directions and any perturbation decays. In the commensurable case, however, the solution may be homogeneous or not in the angle depending on the initial condition, as explained above. As the result of the linear stability analysis Eq. (6) does not depend explicitly 

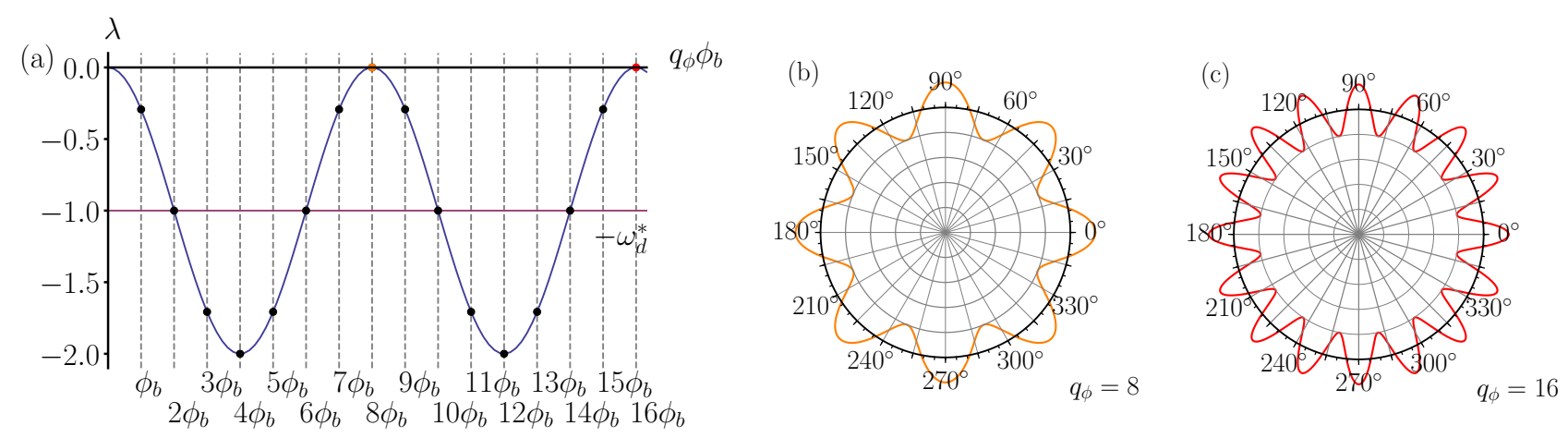

FIG. 2. Linear stability. Panel (a) shows the growth rate $\lambda$ (blue line) of modulations of the density of apices in the angular dimension with wave number $q_{\phi}$ for solutions homogeneous in space. Dashed lines indicate modes compatible with the system size $2 \pi$. The solid orange and red points correspond to the neutral modes shown in panels (b) an (c), respectively.

on the distribution of the directions of growth of the solution, both homogeneous and heterogeneous distributions are stable. There is, however, a set of modes which are neutral, i.e., do neither grow nor decay (Fig. 2). These modes are those with an azimuthal wave number multiple of $2 \pi / \phi_{b}\left(q_{\phi, n}=n 2 \pi / \phi_{b}\right.$ where $n=1,2,3 \ldots)$. For P. Oceanica $q_{\phi, n}=8,16,32 \ldots$. This means that one can redistribute the density of apices among the different directions of growth at no cost, as long as the sum remains constant and the final distribution has the periodicity given by the branching angle. These modes are the result of the breaking of the rotational invariance of the system with respect to the direction of growth. This has very important implications when noise is added to the system, as we are going to discuss in the next section.

\section{THE ROLE OF NOISE}

In this section we study the effects of noise in the case of a branching angle commensurable with $2 \pi$. Here the presence of neutral modes plays a key role in restoring homogeneous density distributions in the growth direction. We consider the case homogeneous in space, while preserving the dependence on the angle including noise. The case of patterns will be discussed in the next section. Thus, we can study the effects of noise with bigger resolutions in the angle at a reasonable computational cost. The ABD model with no space takes the form:

$$
\begin{aligned}
& \partial_{t} n_{a}(\phi, t)= \frac{\omega_{b}}{2}\left[n_{a}\left(\phi+\phi_{b}, t\right)+n_{a}\left(\phi-\phi_{b}, t\right)\right] \\
&-\omega_{d} n_{a}(\phi, t)+\sqrt{\epsilon} \xi(\phi, t), \\
& \partial_{t} n_{s}(t)=-\omega_{d} n_{s}(t)+\frac{v}{\rho} \int_{0}^{2 \pi} n_{a}(\phi, t) d \phi, \\
& \omega_{d}\left(n_{t}\right)=\omega_{d 0}+\left(\kappa-\omega_{d 0}\right)\left(1-e^{-a n_{t}}\right)+b n_{t}^{2} .
\end{aligned}
$$

We have included a white Gaussian noise $\xi(\phi, t)$ with zero mean, variance one, and delta correlated in the angle and in time. $\epsilon$ is the strength of the noise. At first approximation (large average density and small amplitude of the fluctuations), this noise term can model fluctuations in the branching rate. Strictly speaking such fluctuations would lead to a multiplicative noise term as well, but here we are not going to consider this case for simplicity. Explicit noise in the equation of the shoots Eq. (8) is not needed since shoot population is derived directly from the density of apices.

We have performed numerical simulations using a first order pseudospectral method [16,17] to integrate Eqs. (8) and (9) using a time step $\Delta t=1 \times 10^{-3} \omega_{b}^{-1}$. Starting from uniform and nonuniform initial conditions in the angle using two numerical discretizations ( $N=16$ and $N=256$ directions). The uniform initial condition consists of the homogeneous steady state while the nonuniform one includes a periodic modulation with $q_{\phi}=8$ in the growth direction, as the one shown in Fig. 2(b). We have run 1000 realizations of the noise and have computed the average density and the standard deviation of its fluctuations as a function of time for the apices growing in each direction, and for the real part of the corresponding Fourier modes. We use the discrete Fourier transform to compute the amplitude of the modes. The results are shown in Figs. 3 and 4. One should note the last Fourier mode of the discrete Fourier transform encompasses the amplitude of $b_{n}$ and $b_{-n}$ so we use a corrector factor of two for the amplitude of the last mode represented in Fig. 3. As a result the fluctuations in real space are also affected, the effect being more pronounced in Fig. 3(a) where the major contribution to the fluctuations in real space are due to the fluctuations of the last mode. Thus, the prediction for the fluctuations in real space has also been properly corrected.

Numerical simulations starting with uniform initial conditions allow us to analyze the evolution of the fluctuations for the two discretizations used. In both cases, initially, the standard deviation of Fourier modes grows with time. From the linear analysis, we expect damped modes to saturate quickly, following an Ornstein-Uhlenbeck process with zero mean and variance $\frac{\epsilon}{2 \pi} \frac{1-e^{2 \lambda t}}{-2 \lambda}$. However, neutral modes follow a diffusive process of zero mean and a variance that grows in time as $\frac{\epsilon t}{2 \pi}$. Fluctuations in real space are essentially the sum of the fluctuations of all Fourier modes, which are clearly dominated by the neutral modes. Finally, the standard deviation of $n_{a}(\phi)$ is not a perfect diffusive problem for all times and it reaches a stationary value $\sigma=\sqrt{\frac{\Delta \phi}{3}} \frac{N_{a}^{*}}{2 \pi}$, where $\Delta \phi=2 \pi / N$. This can be understood considering the restriction imposed on the stochastic process by the condition of densities being positive. The fluctuations are determined essentially by the neutral 

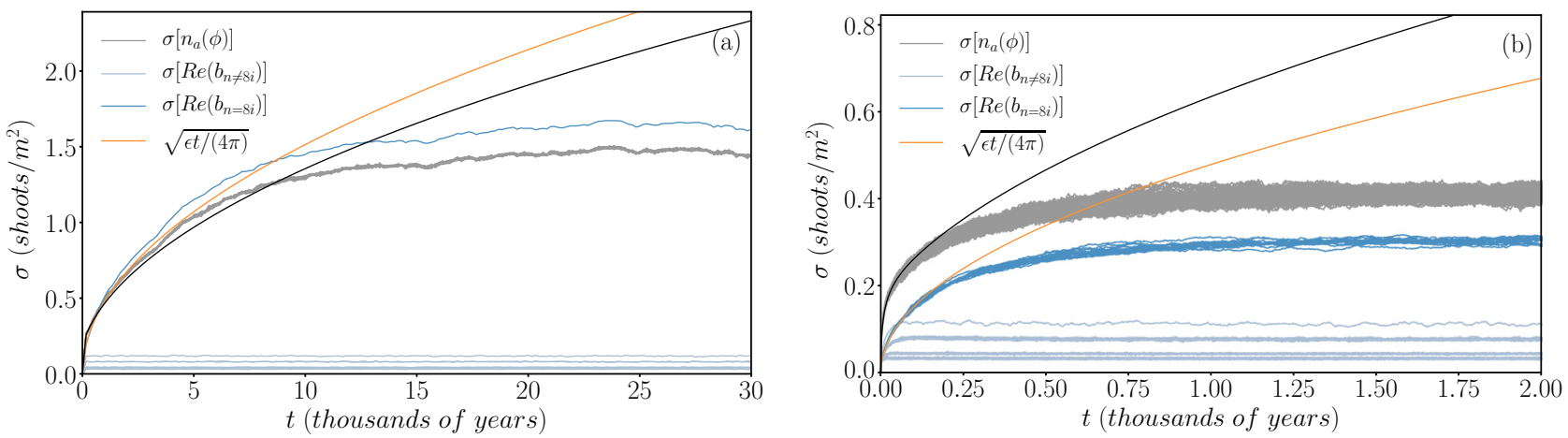

FIG. 3. Evolution of the standard deviation of the density fluctuations in real and Fourier space. Panel (a) corresponds to a numerical discretization $N=16$ directions, while panel (b) $N=256$, using in both cases 1000 realizations of the noise to compute averages. The standard deviations for the fluctuations in each direction $n_{a}(\phi)$ are represented in gray. The real part of the amplitudes of the Fourier modes is shown in dark blue for the neutral modes and in light blue for the damped modes. The orange curve is the theoretical prediction of a diffusive process, while the black line corresponds to the theoretical prediction accounting for all the Fourier modes, neutral and damped. Parameters as described in the caption of Fig. 1 except $\omega_{d 0}=0.03$ year $^{-1}$ and $\epsilon=1.728 \times 10^{-12} \mathrm{~cm}^{-4}$ year $^{-2}$.

modes, which basically experience a random walk in a finite interval, where the amplitude is bounded between $\left[-\frac{N_{a}^{*}}{2 \pi}, \frac{N_{a}^{*}}{2 \pi}\right]$. As fluctuations grow, the probability distribution changes from a Gaussian to a flat distribution and the fluctuations are determined by the size of the interval.

Figure 4 shows the results of numerical simulations starting from a nonuniform initial condition. Figures 4(a) and 4(c) show the density of apices in each growth direction averaged over 1000 noise realizations as a function of time. In the case $N=16$ [Fig. 4(a)], eight growth directions have initially a larger density than the other eight. As time goes by, the difference between the average densities of the two sets of eight growth directions diminishes, until a stationary state is reached with a homogeneous distribution of the density among all growth directions. We notice that the densities of the modes in the same set of eight directions separated by $\phi_{b}$ have almost exactly the same value despite the noise. This is due to the fact that differences between these modes are caused by the excitation of damped modes. As a consequence, the density fluctuation of apices growing in these directions
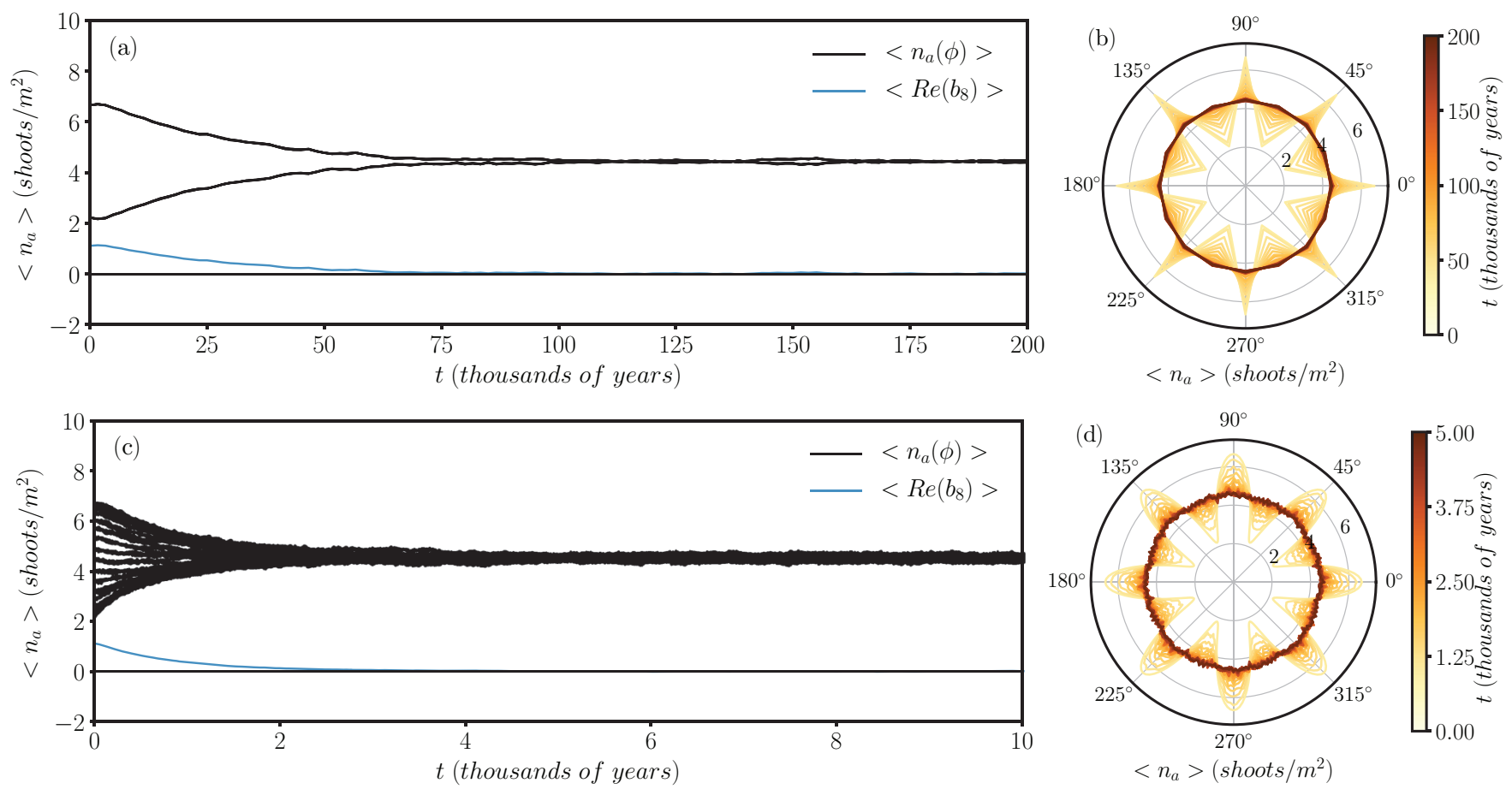

FIG. 4. Time evolution of the distribution of growth directions. Panels (a) and (b) correspond to the numerical discretization of $N=16$ directions of growth. In panel (a) the evolution of the average values of $\left\langle n_{a}(\phi)\right\rangle$ over 1000 realizations are shown for each direction of growth. Two black curves are visible corresponding to the two sets of 8 directions. The blue curve shows the average of the real part of $b_{8}$. Panel (b) shows $\left\langle n_{a}(\phi)\right\rangle$ of each direction, where the color map indicates the time evolution. Panels (c) and (d) show the same as in panels (a) and (b) for $N=256$. Parameters as in Fig. 1 except $\omega_{d 0}=0.03$ year $^{-1}$ and $\epsilon=1.728 \times 10^{-12} \mathrm{~cm}^{-4}$ year $^{-2}$. 
will be highly correlated. Although theoretically such correlations could be measured on the field, in practice such measurements would be extremely complicated to realize and would possible carry very large experimental errors.

Increasing the number of growth directions does not change the picture qualitatively [see Fig. 4(c)]. Starting from an initial condition where the density in the growth direction is modulated, the diffusion of the neutral modes under the action of the noise wipe out these differences on average. In both cases, the average amplitude of neutral mode $b_{8}$ (blue line) decays with time reaching a zero mean. Initially the neutral modes follow a Gaussian distribution with a mean around the initial condition. As time goes trajectories reach the maximum amplitude allowed by the condition of positive densities and the probability distribution changes to a flat distribution with zero mean. Thus, there is a transient in which the mean value decays to zero from the initial value, as can be seen in Fig. 4 for the amplitude $b_{8}$. Otherwise, the rest of the neutral modes with initial zero mean remain with this value along all the time evolution.

These results illustrate how, in general, due to the noise present in real systems, a homogeneous meadow in space will always tend to present a homogeneous density distribution in the growth direction. Any initial perturbation will decay over time due to the effect of the neutral modes and noise.

\section{DISTRIBUTION OF GROWTH DIRECTIONS IN PATTERNS}

For the case of pattern formation (modulational instability of the homogeneous solutions at a finite wave number $q_{x, c}$ ), there is no analytical expression for the growth rate of the perturbations, because the angular part is now coupled to the spatial part. Even so, the linear stability analysis can be performed numerically. One obtains that when the homogeneous solution becomes unstable to patterns, the critical eigenmode has a modulation in the angle. This periodicity does not correspond to any of the neutral modes $q_{\phi}=8,16,32 \ldots$, as in the previous case, but to the periodicity of the system $q_{\phi}=1$, as it can be seen in Fig. 5 .

Analyzing the critical mode the correlation between the spatial pattern and the modulation in the angle is clear. We note that the spatial pattern is shifted according to the direction of growth. The apices growing in the $x$ direction $(\phi=0)$ are shifted to the right, while apices growing in the $-x$ direction $(\phi=\pi)$ are shifted to the left, and equivalently in all the other directions. This heterogeneous distribution in the growth angle associated to the spatial pattern is mainly due to the advection term, which displaces the apices according to the direction of growth. As a result, a stripes pattern does not show a uniform distribution in the angle but it shows a modulation with azimuthal wave number $q_{\phi}=1$.

The stability analysis of a pattern solution including the growth directions is more involved and we do not attempt this analysis in this work. Although the growth rate can not be easily obtained one can demonstrate that solutions $n_{a}^{*}(\vec{x}, \phi)\left[1+\delta n_{a}(\phi)\right]$, where $n_{a}^{*}(\vec{x}, \phi)$ is the stationary pattern, are neutral provided $\delta n_{a}(\phi)$ is a superposition of modes with $q_{\phi, n}=2 \pi n / \phi_{b}$ and $n_{a}^{*}(\vec{x}, \phi) \delta n_{a}(\phi)$ does not change the total density of apices at a given position (see Appendix). To show

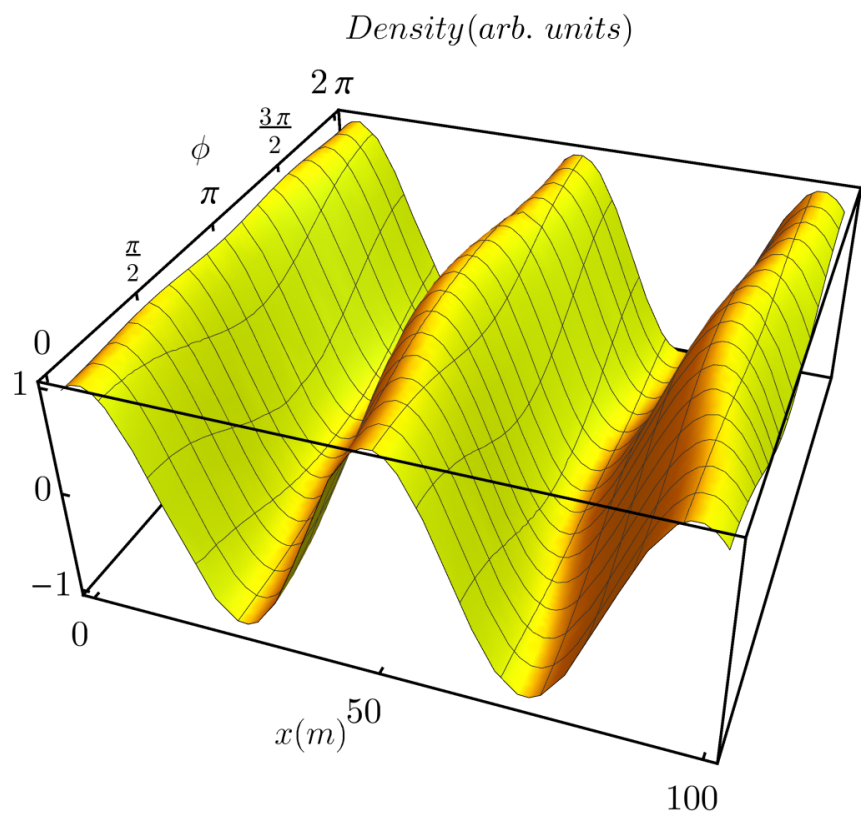

FIG. 5. Critical eigenmode. Eigenstate corresponding to the critical wave number $q_{x, c}=0.1357 \mathrm{~m}^{-1}$ showing an angular dependence with periodicity $2 \pi$. Parameter values as in Fig. 1 .

the existence of neutral modes for branching angles commensurable with $2 \pi$ we use numerical simulations. We integrate Eqs. (1) and (2) using a pseudospectral method [16] with periodic boundary conditions in a square grid with $N_{x}=N_{y}=$ 256 points, spatial discretization $\Delta x=\Delta y=1.066 \mathrm{v} / \omega_{b}$, and $\Delta t=5 \times 10^{-2} \omega_{b}^{-1}$. Note that different directions of growth are considered as different fields on the same spatial grid, which are integrated simultaneously in time. Different solutions can be studied, but we focus on the case of stripes as the results are similar for positive and negative hexagons. We use the pattern of stripes shown in Fig. 1 with $N=16$ growth directions, in such a way that the model is able to describe modulations with azimuthal wave number $q_{\phi}=8$, which corresponds to the first neutral mode. To check the existence of such neutral mode we create three initial patterns with the same total number of apices but different distributions of growth directions and check if modulations in the angle with $q_{\phi}=8$ are neutral or decay with time. Figure 6 shows the stationary state reached from each initial condition. The first initial condition has only the modulation with $q_{\phi}=1$ associated to the growth of the critical mode above threshold. A transverse cut at $y$ constant, represented in orange in Fig. 1, of the density of apices growing in all directions is shown in the bottom row. Notice how the maximum of the density is shifted for each growth direction. The second initial condition consists of all the apices in only one set of 8 growth directions, leaving the densities of the other 8 empty. Such initial condition remains stable as shown in Figs. 6(c) and 6(d). Since the branching is not coupling the two sets of 8 directions, no new apices appear in the empty directions. Finally, we study an initial condition with different densities in both sets of 8 directions, specifically $3 / 4$ and $1 / 4$ of the total density, respectively. Such configuration is also a neutral steady state. From the final solutions in Fig. 6 it becomes clear that there 

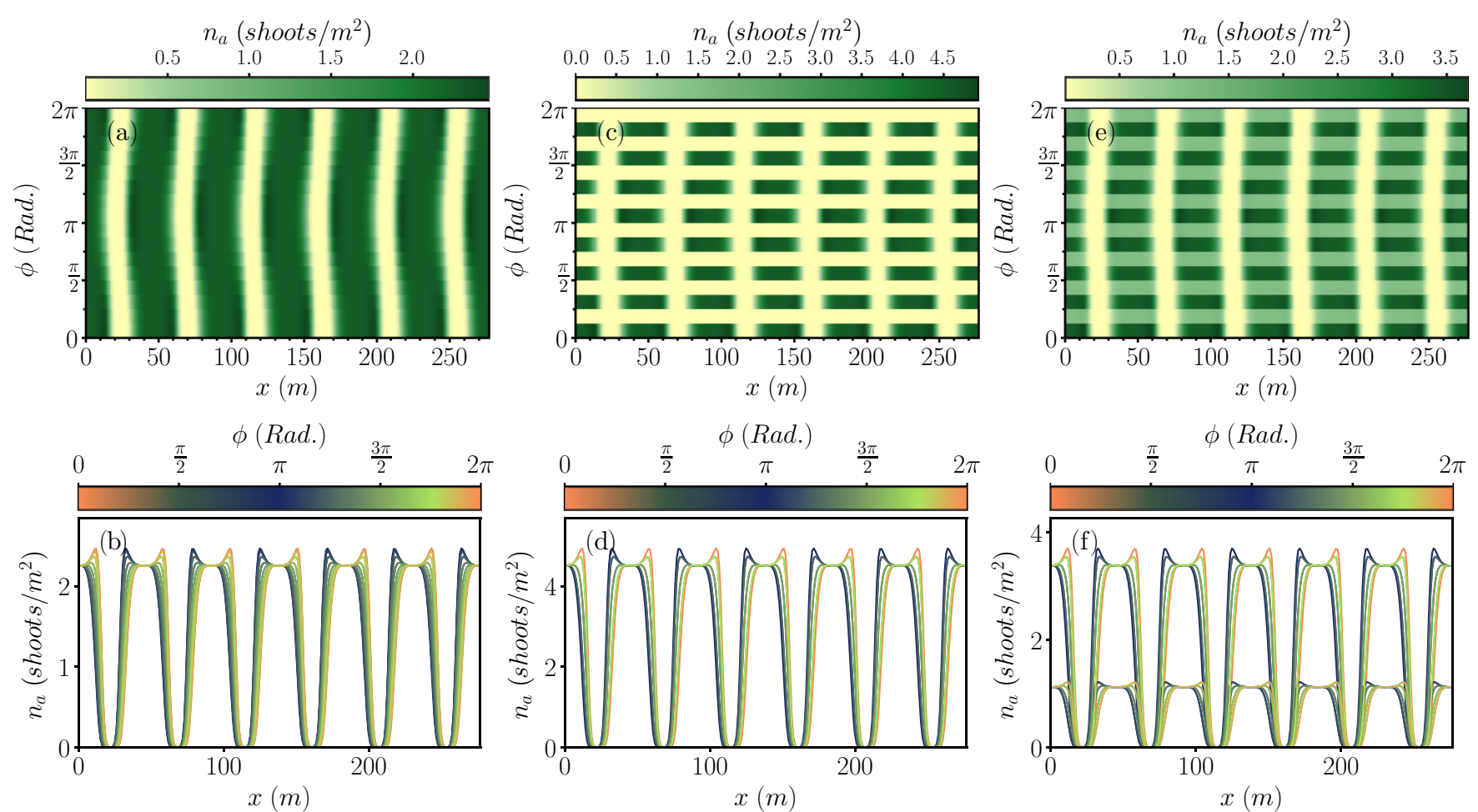

FIG. 6. Growth direction distributions. All panels show the distribution of growth directions for the patterns of Fig. 1, particularly for the cut $y$ constant shown in orange. Panels (a) and (b) correspond to an initial condition where all directions of growth have the same density of apices. Panels (c) and (d) correspond to an initial condition where 8 of the 16 directions of growth have zero density while the other set of eight directions have the same density of apices. Panels (e) and (f) correspond to an initial condition where one set of 8 directions have $1 / 4$ of the total density of apices and the other set the other $3 / 4$ of the density. The same parameters as described in the caption of Fig. 1 have been used.

is a neutral mode that consists in transferring apices from one set of 8 growth directions to the other, as all solutions remain neutrally stable. As explained in the previous section noise will wipe out any such inhomogeneity through the excitation of the neutral modes. The modulation at $q_{\phi}=1$ inherent to the pattern will be, however, robust to noise and it could possibly be measured experimentally.

\section{CONCLUSIONS}

We have studied the role of the branching angle and the discretization of the apices's growth direction in a model for clonal-growth plants. The need to include the growth direction introduces a new effective dimension in the system, increasing considerably the computational cost. Therefore, it is convenient to choose the minimum possible number of growth directions. The straightforward strategy is approximating the branching angle $\phi_{b}$ by the closest angle $\phi_{b}^{\prime}$ commensurable with $2 \pi$, such that we can consistently take $n=2 \pi / \phi_{b}^{\prime}$ growth directions. In this work we have shown that this simple approach is actually justified, and it describes reliably the spatiotemporal dynamics of the meadows.

Using a generic branching angle incommensurable with $2 \pi$, which is the more realistic situation, forces the need of considering a dense discretization of the growth directions; however, it leads, in all cases, to homogeneous distributions. Choosing an angle commensurable with $2 \pi$, however, may lead to solutions not homogeneous in the growth directions, as the coupling through the branching splits the angle grid in decoupled sublattices with $n$ growth directions separated by the branching angle. At the same time, however, this effect introduces neutral modes in the system corresponding precisely to modes that shift population density from one sublattice to another. As a result, when noise is included, the diffusion of these neutral modes wash out any inhomogeneity in the growth direction. On average, then, homogeneous distributions of the growth directions are recovered. This result justifies the use of the minimal discretization associated to the closest angle commensurable with $2 \pi$. Such minimal approach describes only homogeneous density distributions in the angle, which are the only relevant ones in the generic case. Thus, it avoids the possibility of heterogeneous configurations and give the same general results for the space density distribution. Moreover, it describes also suitably the formation of patterns and the induced modulations of the growth direction with $q_{\phi}=1$, well described, for instance by the eight growth directions in the case of $P$. oceanica.

\section{ACKNOWLEDGMENTS}

We acknowledge financial support from Agencia Estatal de Investigación (AEI, Spain) and Fondo Europeo de Desarrollo Regional under Project SuMaEco Grant No. RTI2018095441-B-C22 (AEI/FEDER,UE) and the Spanish State Research Agency, through the Maria de Maeztu Program for units of Excellence in R\&D (MDM-2017-0711). D.R-R. also 
acknowledges the fellowship BES-2016-076264 under the FPI program of MINECO, Spain.

\section{APPENDIX: NEUTRAL MODES FOR STATIONARY PATTERNS}

We devote this Appendix to demonstrating that in the commensurable case, given a stationary solution of the model, which can be heterogeneous in space, like a pattern, due to the presence of neutral modes one can redistribute density of apices in different directions provided the total density at a given position does not change.

Let $n_{s}^{*}(\vec{r}), n_{a}^{*}(\vec{r}, \phi)$ be the stationary solution of Eqs. (1) and (2), as for instance the one represented in Fig. 6. We propose the following ansatz $n_{s}(\vec{r}, t)=n_{s}^{*}(\vec{r})$, $n_{a}(\vec{r}, \phi, t)=n_{a}^{*}(\vec{r}, \phi)\left[1+\delta n_{a}(\phi, t)\right]$, with the condition that $\int_{0}^{2 \pi} n_{a}^{*}(\vec{r}, \phi) \delta n_{a}(\phi, t) d \phi=0$. Thus, the contribution of $\delta n_{a}(\phi, t)$ to the total density at position $\vec{r}$ is zero, and it can only redistribute density in different directions of growth. Hence, introducing the ansatz in Eqs. (1) and (2) we obtain:

$$
\begin{aligned}
n_{a}^{*}(\vec{r}, \phi) \partial_{t} \delta n_{a}(\phi, t) & \\
= & -\omega_{d}\left(n_{t}^{*}\right) n_{a}^{*}(\vec{r}, \phi)-\vec{v}(\phi) \cdot \vec{\nabla} n_{a}^{*}(\vec{r}, \phi) \\
& +\frac{\omega_{b}}{2}\left[n_{a}^{*}\left(\vec{r}, \phi+\phi_{b}\right)+n_{a}^{*}\left(\vec{r}, \phi-\phi_{b}\right)\right] \\
& -\omega_{d}\left(n_{t}^{*}\right) n_{a}^{*}(\vec{r}, \phi) \delta n_{a}(\phi, t)-\vec{v}(\phi) \cdot \vec{\nabla} n_{a}^{*}(\vec{r}, \phi) \delta n_{a}(\phi, t) \\
& +\frac{\omega_{b}}{2}\left[n_{a}^{*}\left(\vec{r}, \phi+\phi_{b}\right) \delta n_{a}\left(\phi+\phi_{b}, t\right)\right. \\
& \left.+n_{a}^{*}\left(\vec{r}, \phi-\phi_{b}\right) \delta n_{a}\left(\phi-\phi_{b}, t\right)\right], \\
& \quad 0=-\omega_{d}\left(n_{t}^{*}\right) n_{s}^{*}(\vec{r})+\frac{v}{\rho} \int_{0}^{2 \pi} n_{a}^{*}(\vec{r}, \phi) d \phi .
\end{aligned}
$$

The first three terms in the right-hand side of Eq. (A1) correspond to the stationary condition and they cancel each other. The fourth and fifth terms are equal to $-\frac{\omega_{b}}{2}\left[n_{a}^{*}\left(\vec{r}, \phi+\phi_{b}\right)+n_{a}^{*}\left(\vec{r}, \phi-\phi_{b}\right)\right] \delta n_{a}(\phi, t)$ according to the stationary condition. Equation (A2) corresponds to the stationary condition for Eq. (2). Thus, one obtains the following expression:

$$
\begin{aligned}
n_{a}^{*}(\vec{r}, \phi) \partial_{t} \delta n_{a}(\phi, t) \\
=-\frac{\omega_{b}}{2}\left[n_{a}^{*}\left(\vec{r}, \phi+\phi_{b}\right)+n_{a}^{*}\left(\vec{r}, \phi-\phi_{b}\right)\right] \delta n_{a}(\phi, t) \\
\quad+\frac{\omega_{b}}{2}\left[n_{a}^{*}\left(\vec{r}, \phi+\phi_{b}\right) \delta n_{a}\left(\phi+\phi_{b}, t\right)\right. \\
\left.\quad+n_{a}^{*}\left(\vec{r}, \phi-\phi_{b}\right) \delta n_{a}\left(\phi-\phi_{b}, t\right)\right] .
\end{aligned}
$$

One can write $n_{a}^{*}(\vec{r}, \phi)=\sum_{q_{\phi}} b_{q_{\phi}}^{*}(\vec{r}) e^{i q_{\phi} \phi}$ and $\delta n_{a}(\phi, t)=$ $\sum_{q_{\phi}^{\prime}} \delta b_{q_{\phi}^{\prime}}(t) e^{i q_{\phi}^{\prime} \phi}$ as a Fourier series and substitute in Eq. (A3). Rearranging terms with the same exponential term $q=q_{\phi}+$ $q_{\phi}^{\prime}$ one obtains a set of ordinary differential equations labeled by $q \in \mathbb{Z}$ of the following form:

$$
\begin{aligned}
\sum_{q_{\phi}^{\prime}} b_{q-q_{\phi}^{\prime}}^{*}(\vec{r}) \partial_{t} \delta b_{q_{\phi}^{\prime}}(t)= & \sum_{q_{\phi}^{\prime}} \omega_{b}\left\{\cos \left(q \phi_{b}\right)\right. \\
& \left.-\cos \left[\left(q-q_{\phi}^{\prime}\right) \phi_{b}\right]\right\} b_{q-q_{\phi}^{\prime}}^{*}(\vec{r}) \delta b_{q_{\phi}^{\prime}}(t) .
\end{aligned}
$$

The eigenvalues of the problem are difficult to obtain from Eq. (A4), and they may not have an analytical expression; however, a more simple strategy is to impose the condition that the modes of the perturbation are those with $q_{\phi, n}^{\prime}=\frac{2 \pi}{\phi_{b}} n$, which makes the right-hand side of Eq. (A4) zero. Hence, these modes are neutral independently of the stationary solution, meaning one can redistribute density in different directions as long as it is periodic with $\phi_{b}$ and does not change the total density of apices.
[1] M. A. Hemminga and C. M. Duarte, Seagrass Ecology (Cambridge University Press, Cambridge, 2000).

[2] C. M. Duarte, T. Sintes, and N. Marbà, J. Appl. Ecol. 50, 1341 (2013).

[3] N. Marbà, E. Díaz-Almela, and C. M. Duarte, Biol. Conserv. 176, 183 (2014).

[4] M. Waycott, C. M. Duarte, T. J. B. Carruthers, R. J. Orth, W. C. Dennison, S. Olyarnik, A. Calladine, J. W. Fourqurean, K. L. Heck, A. R. Hughes, G. A. Kendrick, W. J. Kenworthy, F. T. Short, and S. L. Williams, Proc. Natl. Acad. Sci. USA 106, 12377 (2009).

[5] D. Ruiz-Reynés, D. Gomila, T. Sintes, E. Hernández-García, N. Marbà, and C. M. Duarte, Sci. Adv. 3, e1603262 (2017).

[6] T. Sintes, N. Marbà, C. M. Duarte, and G. A. Kendrick, Oikos 108, 165 (2005).

[7] G. A. Kendrick, N. Marbà, and C. M. Duarte, Estuar. Coast. Shelf Sci. 65, 717 (2005).

[8] M. L. Cain, J. Ecol. 78, 27 (1990).
[9] A. D. Bell and P. B. Tomlinson, Botan. J. Linn. Soc. 80, 125 (1980).

[10] J. Piqueras, L. Klimeš, and P. Redbo-Torstensson, Plant Ecol. 141, 117 (1999).

[11] B. Oborny, C. Mony, and T. Herben, Ecol. Model. 234, 3 (2012).

[12] O. Lejeune and M. Tlidi, J. Veg. Sci. 10, 201 (1999).

[13] J. von Hardenberg, E. Meron, M. Shachak, and Y. Zarmi, Phys. Rev. Lett. 87, 198101 (2001).

[14] M. Rietkerk and J. van de Koppel, Trends Ecol. Evol. 23, 169 (2008).

[15] T. Sintes, N. Marbà, and C. M. Duarte, Estuaries Coasts 29, 72 (2006).

[16] R. Montagne, E. Hernández-García, A. Amengual, and M. San Miguel, Phys. Rev. E 56, 151 (1997).

[17] R. Toral and P. Colet, Stochastic Numerical Methods: An Introduction for Students and Scientists (John Wiley \& Sons, New York, 2014). 